\title{
Modelo de Intervención de Riesgos Psicosociales en la Red Asistencial de Salud
}

\author{
INTERVENTION MODEL OF PSYCHOSOCIAL RISKS IN PUBLIC HEALTH FACILITIES
}

\author{
Cristian Villarroel ${ }^{1}$, Carolina Busco R. ${ }^{1}$, Belén Neira L. ${ }^{3}$, André Ensignia E. ${ }^{4}$ Pablo Durán V. ${ }^{1}$ \\ 1. Departamento de Salud Ocupacional, Ministerio de Salud, Chile. \\ 2. Docente Facultad de Ingeniería, Universidad Diego Portales. \\ 3. Ingeniera Civil Industrial. \\ 4. Subdirección de Gestión y Desarrollo de Personas. Servicio Civil, Chile.
}

\begin{abstract}
RESUMEN
En Chile, la normativa exige que todas las empresas e instituciones evalúen y gestionen los riesgos psicosociales en sus lugares de trabajo. Uno de los sectores donde se produce la mayor cantidad de casos de patologías de salud mental de origen laboral es, justamente, en el ámbito de la atención en salud.

Motivados en esta situación, el presente estudio abordó los datos obtenidos en dos establecimientos públicos de salud y generó un tratamiento estadístico capaz de identificar los principales factores de riesgo para la población estudiada.
\end{abstract}

(Villarroel C, Busco C, Neira B, Ensignia A, Durán P, 2018. Modelo de Intervención de Riesgos Psicosociales en la Red Asistencial de Salud. Cienc Trab. May-Ago; 20 [62]: 76-79).

Palabras clave: RIESGO PSICOSOCIAL, SALUD LABORAL, SALUD PÚBLICA.

\section{ABSTRACT}

In Chile, the regulations require that all companies and institutions evaluate and manage psychosocial risks at their workplaces. One of the work areas where the greatest number of cases of mental health pathologies related to work occurs, is precisely in the field of health care.

Motivated by this situation, this study addressed the data obtained in two Public Health Facilities to generate a statistical treatment capable of identify the main risk factor's for the population studied.

Key words: PSYCHOSOCIAL RISK, OCCUPATIONAL HEALTH, PUBLIC HEALTH.

\section{INTRODUCCION}

Según la OIT y la OMS, la existencia de factores psicosociales en el trabajo no solo influye en la salud de las personas, sino también en el rendimiento y productividad de la institución. Para su análisis, existen diversas dimensiones que deben ser consideradas, tales como la forma en que se organiza el trabajo, el contexto en el que se desarrollan las tareas, el perfil de los trabajadores/as y el sector productivo en el cual se generan los bienes o servicios.

En Chile, los riesgos psicosociales laborales se encuentran regulados por el Ministerio de Salud y son definidos como aquellas "situaciones y condiciones inherentes al trabajo y relacionadas al tipo de organización, al contenido y la ejecución de la tarea que tienen la capacidad de afectar, en forma positiva o negativa, el bienestar y la salud (física, psíquica o social) del trabajador y sus condiciones de trabajo". ${ }^{1}$

Correspondencia / Correspondence:

Cristián Villarroel P.

e-mail: cvillarroelp@gmail.com

Recibido: 11 de Abril de 2018 / Aceptado: 25 de Junio de 2018
Esta definición permite, por una parte, establecer un marco normativo para la medición y gestión del riesgo y, por otra, la posibilidad de abrir un diálogo social sobre el tipo de organización del trabajo esperado, sea en un contexto público o privado. A la fecha, más de ochocientos mil trabajadores y trabajadoras han formado parte de los procesos de evaluación e intervención de riesgos psicosociales en sus lugares de trabajo (16\% del total de trabajadores formales sujetos a los deberes y derechos de la Ley 16.744 y 19.345 que regulan los accidentes del trabajo y las enfermedades profesionales en Chile), abriendo la posibilidad de generar datos que permiten analizar, evaluar, comparar y proponer mejoras a nuestra cultura laboral.

En Chile, el 76\% de la población se atiende en la red asistencial pública de salud, convirtiendo a este sector en un foco de atención para los medios de comunicación.

A su vez, la ciudadanía ha incrementado la exigencia de respuestas oportunas y satisfactorias de las instituciones públicas hacia los usuarios, lo que impacta y tensiona el quehacer de las instituciones y el desempeño de sus trabajadores, quienes deben reaccionar y adaptarse a un contexto cada vez más desafiante. La naturaleza del trabajo de las organizaciones de la salud genera un ambiente laboral que la evidencia internacional califica de "estresante". En este contexto, la evaluación de riesgos psicosociales y su posterior análisis en el ámbito de la salud se presenta como una oportunidad para revisar formas de organización del trabajo. 
El presente estudio representa un esfuerzo por sistematizar la experiencia de evaluación de riesgos psicosociales en dos establecimientos del área pública de la salud, con el fin de proponer un modelo de análisis que mejore la efectividad de las intervenciones.

\section{OBJETIVOS DE LA INVESTIGACIÓN}

A nivel mundial, el estrés se ha transformado en el segundo problema de salud más frecuente relacionado con el trabajo, siendo la molestia de espalda el primero. ${ }^{2}$ Este estrés ligado al trabajo es causado principalmente por riesgos psicosociales, que pueden vincularse con el diseño y la gestión organizacional, lo que se suma naturalmente a los riesgos físicos. ${ }^{3}$ En Chile, la principal causa de licencias médicas del ámbito laboral están dadas por la salud mental ${ }^{4}$, donde se reconoce que el ambiente laboral constituye un factor importante al momento de referirse a la salud mental de una persona.

Este estudio pretende identificar aspectos de la organización que entorpecen el bienestar del ambiente laboral y de sus trabajadores, otorgando una relevancia central a elementos asociados al apoyo social y calidad de liderazgo. En otras palabras, la interacción entre las personas, sean estas pares o superiores, jugaría un rol central a la hora de explicar la probabilidad de los trabajadores de sufrir riesgos psicosociales en su lugar de trabajo. Esta interrelación entre el trabajador y la organización se define de acuerdo a un "contrato psicosocial", el cual hace referencia al "conjunto de compensaciones que la persona (o grupo) espera de la organización a cambio de sus contribuciones y al conjunto de compensaciones que la organización espera de la persona a cambio de sus contribuciones". 5

A su vez, se estima que algunas variables sociodemográficas, así como algunos aspectos normativos o estructurales de la organización, estarían siendo relevantes a la hora de explicar la incidencia de estos riesgos al interior de la organización.

En este contexto, el objetivo del presente estudio es identificar la existencia y centralidad de algunos factores de riesgo psicosocial en los establecimientos públicos del sector salud.

La hipótesis plantea que "los riesgos psicosociales se encuentran explicados por la dimensión 'Apoyo social en la empresa y calidad del liderazgo', así como por variables sociodemográficas y condiciones de empleo, cuyas implicancias son distintas de acuerdo al nivel de complejidad de cada establecimiento".

El tipo y nivel de complejidad de un establecimiento es un antecedente significativo en materia de riesgo psicosocial en sus funcionarios, dado que se vincula con una serie de variables relevantes, tales como: prevalencia de un trabajo asistencial por sobre el de tipo administrativo, existencia de sistemas de turnos, así como la complejidad en el volumen y la estructura de la organización.

Para efectos de este estudio, se abordan dos tipos de establecimiento: uno de baja complejidad y otro de alta complejidad. Un establecimiento de baja complejidad es aquel que tiene la función de acercar la salud a la población, principalmente en zonas extremas o en presencia de ruralidad, dando cobertura a toda la población de su territorio en prestaciones de menor complejidad de salud. A su vez, los establecimientos de alta complejidad entregan servicios destinados a la recuperación, rehabilitación y cuidados paliativos de personas enfermas y de colaborar en las actividades de fomento y protección, mediante acciones fundamentalmente de atención cerrada o ambulatoria.
Tabla 1.

Distribución de la muestra.

\begin{tabular}{lcccc} 
Muestra & $\begin{array}{c}\text { Dotación } \\
\text { Femenina }\end{array}$ & $\begin{array}{c}\text { Dotación } \\
\text { Masculina }\end{array}$ & Total (N) & $\begin{array}{c}\% \\
\text { Participación }\end{array}$ \\
\hline $\begin{array}{l}\text { Establecimiento de } \\
\text { Alta Complejidad }\end{array}$ & 387 & 190 & 577 & $71,58 \%$ \\
\hline $\begin{array}{l}\text { Establecimiento de } \\
\text { Baja Complejidad }\end{array}$ & 58 & 29 & 87 & $96,55 \%$
\end{tabular}

\section{MÉTODO}

El estudio utilizó un diseño de tipo transversal, cuantitativo, descriptivo y correlacional. La muestra seleccionada (ver Tabla № 1) es de carácter no probabilístico y estratégico, constituida por 664 trabajadores y trabajadoras pertenecientes a dos establecimientos de salud. El instrumento utilizado corresponde al "Cuestionario de evaluación de riesgos psicosociales en el trabajo, SUSESO/ISTAS21, versión completa”. Este cuestionario es una adaptación de El Copenhagen Psicosocial Questionnaire (COPSOQ-ISTAS21) ${ }^{6}$, que se basa en dos modelos de riesgos psicosociales: el desbalanceesfuerzo-recompensa de Siegrist y el modelo demanda-controlapoyo-social de Karasek y Theorell.

El análisis de los datos se realizó mediante el Software IBM SPSS Statistics versión 20, desarrollando correlaciones bivariadas, con el fin de medir la fuerza o el grado de asociación lineal entre variables. Se establecen cuatro modelos de regresión lineal múltiple por cada establecimiento y su nivel de complejidad, utilizando una significancia de 0,05 para el rechazo de la hipótesis nula.

La regresión lineal múltiple tiene como finalidad predecir el valor promedio de una variable sobre la base de valores fijos de otras variables. ${ }^{7}$ En otras palabras, este tipo de regresión es "más adecuado para un análisis ceteris paribus debido a que permite controlar de manera explícita muchos otros factores que afectan en forma simultánea a la variable dependiente" ${ }^{9}$ de acuerdo a la siguiente demostración:

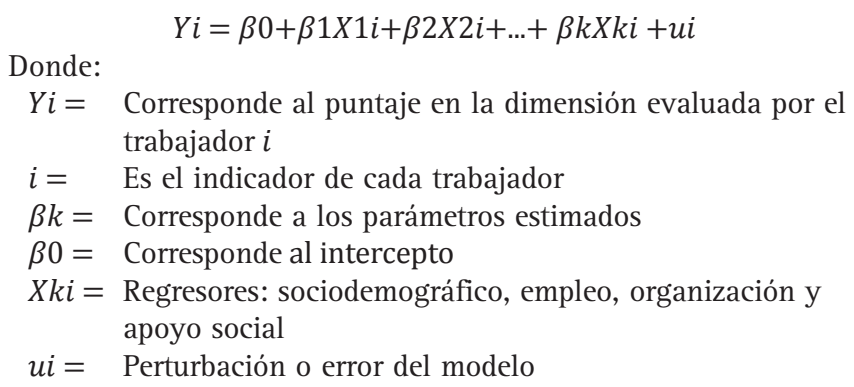

Los modelos de regresión lineal fueron estimados mediante el método de Mínimos Cuadrados Ordinarios (MCO). Para ello, es necesario que se cumplan una serie de supuestos. ${ }^{7}$ El modelo clásico de regresión lineal, además de contemplar los supuestos de Gauss-Markov, incluye el supuesto clásico de normalidad de los errores. ${ }^{8}$

\section{RESULTADOS}

El análisis estadístico de la información arrojó dos modelos donde se establece como factor principal explicativo a la dimensión "Apoyo social en la empresa y calidad del liderazgo", que corresponde a una de las dimensiones de riesgo psicosocial abordadas por el Cuestionario SUSESO/ISTAS21 validado para realidad laboral chilena. 
Matriz de identificación de riesgos psicosociales en un establecimiento de alta especialidad.

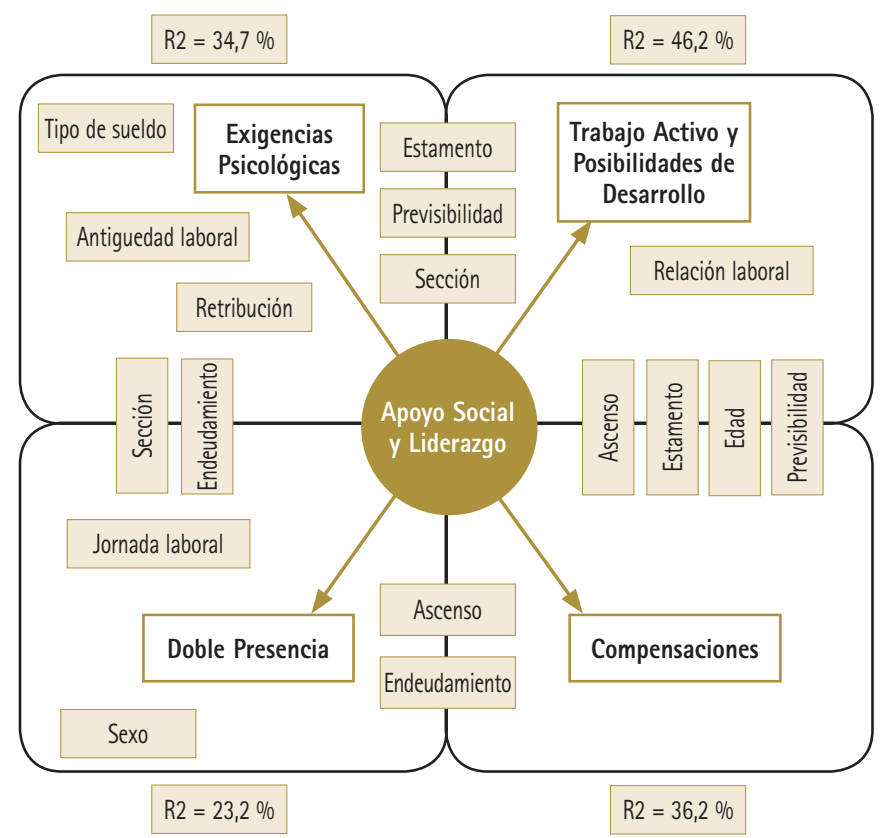

A continuación, se presentan los modelos finales de cada una de las muestras, elaborados a partir del cruce de las regresiones lineales de cada dimensión y sus respectivas bondades de ajuste.

En la matriz resultante, el factor "Apoyo social en la empresa y calidad del liderazgo" constituye el eje del modelo, desde donde es posible explicar el comportamiento de los otros factores de riesgo.

Existen variables compartidas entre dichos factores (sección, nivel de endeudamiento, ascenso, entre otros), entregando elementos de análisis; sin embargo, la centralidad está dada por las formas que adquiere el liderazgo y la repercusión que tiene en el riesgo autopercibido de trabajadoras y trabajadores.

$\mathrm{Al}$ igual que en el modelo anterior, la dimensión "Apoyo social en la empresa y calidad del liderazgo" explica significativamente en la totalidad de las variables dependientes.

Si bien existen variables que se comparten entre dimensiones, se debe destacar aquellas que explican de manera única a cada uno de sus modelos; esto se puede visualizar en aquellas que se encuentran en un solo cuadrante, explicando a su respectiva dimensión y no en medio de dos, como son las variables "compartidas".

\section{DISCUSIÓN}

Para efectos de este artículo, se entregan algunos elementos para enriquecer la discusión sobre la medición de riesgos psicosociales con el fin de aportar antecedentes para futuras reflexiones e intervenciones en el ámbito de la salud y otros sectores de la producción.
Matriz de identificación de riesgos psicosociales en un establecimiento de baja complejidad.

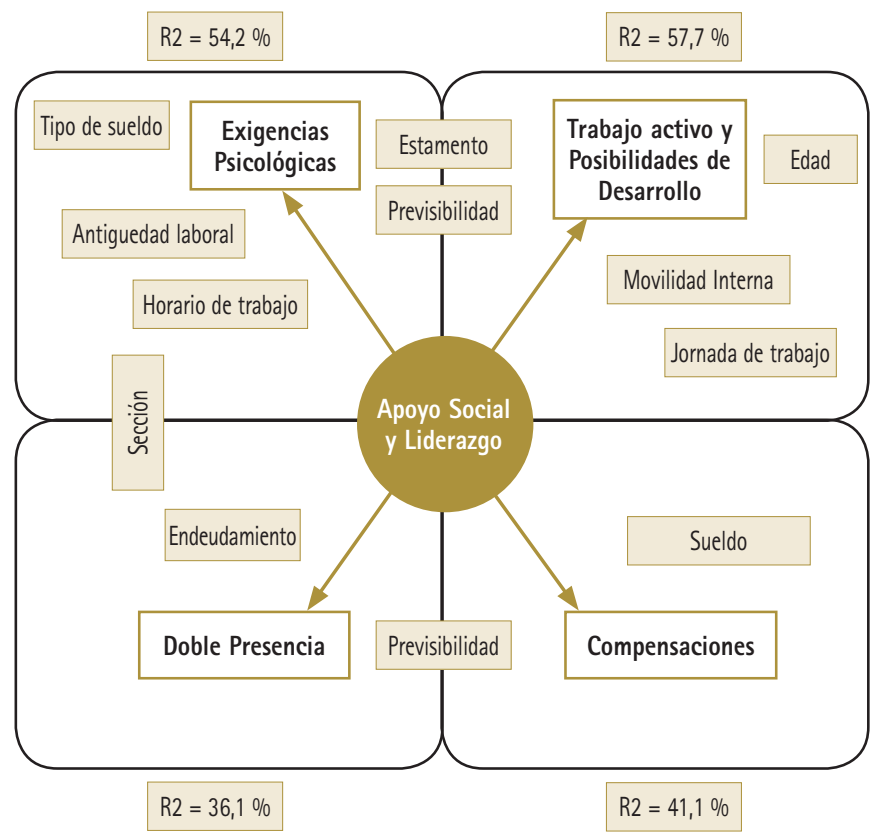

Lo primero es señalar la centralidad de la dimensión "Apoyo social en la empresa y calidad del liderazgo", que media y modela el comportamiento de las otras dimensiones y variables en estudio. Ello adquiere una relevancia a la hora de las intervenciones, junto a un principio de prioridad y economía a la hora de focalizar las acciones e intervenciones.

Desde el punto de vista de la gestión en establecimientos de salud públicos, el eje en el liderazgo conlleva a focalizar la mitigación de los riesgos psicosociales en aquellos aspectos y puestos claves dentro de la organización, que resultan más sensibles en la producción hospitalaria.

Respecto de las variables sociodemográficas, cabe destacar que todas fueron significativas en los modelos elaborados. La edad es considerada una variable que explica a los modelos de "Trabajo activo y desarrollo de habilidades", tanto para la muestra de alta complejidad como la de baja complejidad.

Los datos confirman la evidencia teórica que señala que los jóvenes y las mujeres son aquellas subpoblaciones de mayor exposición a los riesgos psicosociales.

En términos de estamentos, los modelos identifican coincidentemente a los auxiliares y técnicos como aquellos trabajadores con mayor percepción de riesgo en la dimensión "Trabajo activo y desarrollo de habilidades".

En las condiciones de empleo, las variables vinculadas a las condiciones de empleo adquieren especial relevancia en la dimensión "Doble presencia". A su vez, el horario de trabajo es una variable significativa para el modelo de baja complejidad, no así para el de alta complejidad. La variable de previsibilidad explica todas las dimensiones de riesgo psicosocial en un establecimiento de baja complejidad. 


\section{REFERENCES}

1. Gobierno de Chile. Ministerio de Salud. Protocolo de Vigilancia de Riesgos Psicosociales en el Trabajo [en línea]. Santiago de Chile: MINSAL; 2017 [citado abr 2018]. 68 p. Disponible en: http://www.minsal.cl/portal/url/item/e0397723 56757886e040010165014a72.pdf.

2. Milczarek $M$, Brun E. Expert forecast on emerging psychosocial risks related to occupational safety and health. Luxembourg: Office for Official Publications of the European Communities; 2007.

3. Agencia Europea para la Seguridad y Salud en el Trabajo. Investigación sobre el estrés relacionado con el trabajo [en línea]. Luxemburgo: Oficina de Publicaciones Oficiales de las Comunidades Europeas; 2005 [citado may 2018]. 167 p. Disponible en: https://osha.europa.eu/es/tools-andpublications/publications/ reports/203/view.

4. Superintendencia de Seguridad Social. Informe anual 2017; estadísticas de seguridad social [en línea]. Santiago de Chile: SUSESO; 2018 [citado may 2018]. Disponible en: http://www.suseso.gob.cl/607/articles-496701_archivo_01.pdf.

5. Peiró $G$, Sánchez $P$, Corbellas $C$ et al. Sintomatología psicológica relatada por pacientes con trastornos gastrointestinales funcionales. Revista Electrónica de Motivación y Emoción (REME) [en línea]. 2006 [citado may 2018]; 9(2324):1-33. Disponible en: https://kipdf.com/sintomatologia-psicologica-relatada-por-pacientes-con-trastornos-gastrointestina_5b165ce67f8b9a3309 8b45f9.html.

6. Kristensen TS., Moncada S, Llorens C. NTP 703: El método COPSO0 (ISTAS21, PSOCAT21) de evaluación de riesgos psicosociales [en línea]. Madrid: Instituto Nacional de Higiene y Seguridad en el Trabajo; 200? [citado abr 2018]. Disponible en: http://www.insht.es/InshtWeb/Contenidos/Documentacion/FichasTecnicas/NTP/Ficheros/701a750/ntp_703.pdf

7. Gujarati, DN. Econometría básica. 3a ed. Bogotá: McGraw-Hill; 1997.

8. Cabello M. Econometría Fácil: Una guia simple a las complejidades del análisis de regresión [e-book en línea]. San Francisco: SCRIBD; 2016 [citado may 2018]. Disponible en: https://es.scribd.com/document/323777836/1-Intro-MCO.

9. Wooldridge JM. Introductory Econometrics. 4th ed. Mason: South-Western Cengage Learning; 2009. 